\title{
PARABOLIC FRACTIONAL MAXIMAL AND INTEGRAL OPERATORS WITH ROUGH KERNELS IN PARABOLIC GENERALIZED MORREY SPACES
}

\author{
VAgiF S. GuliyeV and Aydin S. Balakishiyev
}

Abstract. Let $P$ be a real $n \times n$ matrix, whose all the eigenvalues have positive real part, $A_{t}=t^{P}$, $t>0, \gamma=\operatorname{tr} P$ is the homogeneous dimension on $\mathbb{R}^{n}$ and $\Omega$ is an $A_{t}$-homogeneous of degree zero function, integrable to a power $s>1$ on the unit sphere generated by the corresponding parabolic metric. We study the parabolic fractional maximal and integral operators $M_{\Omega, \alpha}^{P}$ and $I_{\Omega, \alpha}^{P}, 0<\alpha<\gamma$ with rough kernels in the parabolic generalized Morrey space $\mathscr{M}_{p, \varphi, P}\left(\mathbb{R}^{n}\right)$. We find conditions on the pair $\left(\varphi_{1}, \varphi_{2}\right)$ for the boundedness of the operators $M_{\Omega, \alpha}^{P}$ and $I_{\Omega, \alpha}^{P}$ from the space $\mathscr{M}_{p, \varphi_{1}, P}\left(\mathbb{R}^{n}\right)$ to another one $\mathscr{M}_{q, \varphi_{2}, P}\left(\mathbb{R}^{n}\right), 1<p<q<\infty, 1 / p-1 / q=\alpha / \gamma$, and from the space $\mathscr{M}_{1, \varphi_{1}, P}\left(\mathbb{R}^{n}\right)$ to the weak space $W \mathscr{M}_{q, \varphi_{2}, P}\left(\mathbb{R}^{n}\right), 1 \leqslant q<\infty, 1-1 / q=$ $\alpha / \gamma$. We also find conditions on $\varphi$ for the validity of the Adams type theorems $M_{\Omega, \alpha}^{P}, I_{\Omega, \alpha}^{P}$ : $\mathscr{M}_{p, \varphi \frac{1}{p}, P}\left(\mathbb{R}^{n}\right) \rightarrow \mathscr{M}_{q, \varphi^{\frac{1}{q}}, P}\left(\mathbb{R}^{n}\right), 1<p<q<\infty$.

Mathematics subject classification (2010): 42B20, 42B25, 42B35.

Keywords and phrases: Parabolic fractional maximal function, parabolic fractional integral, parabolic generalized Morrey space.

\section{REFERENCES}

[1] D. R. Adams, A note on Riesz potentials, Duke Math. 42 (1975), 765-778.

[2] A. Akbulut, V. S. Guliyev, R. Mustafayev, On the boundedness of the maximal operator and singular integral operators in generalized Morrey spaces, Math. Bohem. 137, 1 (2012), 27-43.

[3] O. V. Besov, V. P. IL'IN, P. I. Lizor Kin, The $L_{p}$-estimates of a certain class of non-isotropically singular integrals, (Russian) Dokl. Akad. Nauk SSSR 169 (1966), 1250-1253.

[4] V. I. Burenkov, H. V. Guliyev, And V. S. Guliyev, Necessary and sufficient conditions for boundedness of the fractional maximal operators in the local Morrey-type spaces, J. Comput. Appl. Math. 208, 1 (2007), 280-301.

[5] V. Burenkov, A. Gogatishvili, V. S. Guliyev, R. Mustafayev, Boundedness of the fractional maximal operator in local Morrey-type spaces, Complex Var. Elliptic Equ. 55, 8-10 (2010), 739-758.

[6] V. Burenkov, A. Gogatishvili, V. S. Guliyev, R. Mustafayev, Boundedness of the Riesz potential in local Morrey-type spaces, Potential Anal. 35, 1 (2011), 67-87.

[7] A. P. CAldereon, A. TORChins Ky, Parabolic maximal function associated with a distribution, I., Advances in Math. 16 (1975), 1-64.

[8] R. R. COIFMAN, G. WeIss, Analyse harmonique non-commutative sur certains espaces homogènes, (French) Étude de certaines intégrales singuliàres, Lecture Notes in Mathematics, Vol. 242. SpringerVerlag, Berlin-New York, 1971.

[9] E. B. FABES, N. RIVÈrE, Singular integrals with mixed homogeneity, Studia Math. 27 (1966), 19-38.

[10] G. B. Folland, E. M. Stein, Hardy Spaces on Homogeneous Groups, Math. Notes, 28, Princeton Univ. Press, Princeton, 1982.

[11] Y. Ding, D. YANG, Z. ZHOU, Boundedness of sublinear operators and commutators on $L^{p, \omega}\left(\mathbb{R}^{n}\right)$, Yokohama Math. J. 46 (1998), 15-27. 
[12] M. GIAQUINTA, Multiple integrals in the calculus of variations and nonlinear elliptic systems, Princeton Univ. Press, Princeton, NJ, 1983.

[13] V. S. GULIYEV, Integral operators on function spaces on the homogeneous groups and on domains in $\mathbb{R}^{n}$, Doctor's degree dissertation, Mat. Inst. Steklov, Moscow, 1994, 329 pp. (in Russian).

[14] V. S. GuliYev, Function spaces, integral operators and two weighted inequalities on homogeneous groups. Some applications, Casioglu, Baku, 1999, 332 pp. (in Russian).

[15] V. S. GuliYev, Boundedness of the maximal, potential and singular operators in the generalized Morrey spaces, J. Inequal. Appl. 2009, Art. ID 503948, 1-20.

[16] V. S. Guliyev, R. Mustafayev, Boundedness of the anisotropic maximal and anisotropic singular integral operators in generalized Morrey spaces, Acta Mathematica Sinica (English series) 27, 12 (2011), 2361-2370.

[17] V. S. Guliyev, S. S. Aliyev, T. Karaman, And P. S. Shukurov, Boundedness of sublinear operators and commutators on generalized Morrey Space, Integral Equat. Oper. Theor. 71, 3 (2011), $327-355$.

[18] V. S. GuliYEv, Generalized local Morrey spaces and fractional integral operators with rough kernel, J. Math. Sci. (N.Y.) 193, 2 (2013), 211-227.

[19] V. S. GuliYev, L. Softova, Global regularity in generalized Morrey spaces of solutions to nondivergence elliptic equations with VMO coefficients, Potential Anal. 38, 4 (2013), 843-862.

[20] A. Maugeri, D. K. Palagachev, L. G. Softova, Elliptic and Parabolic Equations with Discontinuous Coefficients, Wiley-VCH, Berlin 2000.

[21] T. Mizuhara, Boundedness of some classical operators on generalized Morrey spaces, In: Harmonic Analysis, Proc. Conf., Sendai/Jap. 1990, ICM-90 Satell. Conf. Proc., pp. 183-189 (1991).

[22] C. B. Morrey, On the solutions of quasi-linear elliptic partial differential equations, Trans. Amer. Math. Soc. 43 (1938), 126-166.

[23] B. Mukenhoupt And R. L. Wheeden, Weighted norm inequalities for singular and fractional integral, Trans. Amer. Math. Sco. 161 (1971), 249-258.

[24] E. NAKAI, Hardy-Littlewood maximal operator, singular integral operators and the Riesz potentials on generalized Morrey spaces, Math. Nachr. 166 (1994), 95-103.

[25] E. NAKAI, The Campanato, Morrey and Hölder spaces on spaces of homogeneous type, Studia Math. 176, 1 (2006), 1-19.

[26] L.-E. Persson, N. SAmko, Weighted Hardy and potential operators in the generalized Morrey spaces, J. Math. Anal. Appl. 377, 2 (2011), 792-806.

[27] E. M. Stein, Harmonic Analysis: Real Variable Methods, Orthogonality and Oscillatory Integrals, Princeton Univ. Press, Princeton NJ, 1993.

[28] E. M. Stein And S. Wainger, Problems in harmonic analysis related to curvature, Bull. Amer. Math. Soc. 84 (1978), 1239-1295.

[29] Na Wei, Pengcheng Niu, Sufang Tang, Maochun Zhu, Estimates in generalized Morrey spaces for nondivergence degenerate elliptic operators with discontinuous coefficients, Rev. R. Acad. Cienc. Exactas Fís. Nat. Ser. A Math. RACSAM 106, 1 (2012), 1-33.

[30] Lin Zhang, Yin Sheng Jiang, Jiang Zhou, Parabolic equations with VMO coefficients in generalized Morrey spaces, Acta Math. Sin. (Engl. Ser.) 26, 1 (2010), 117-130. 